\title{
COHOMOLOGY FOR A GROUP OF DIFFEOMORPHISMS OF A MANIFOLD PRESERVING AN EXACT FORM.
}

\author{
MARK LOSIK, PETER W. MICHOR
}

\begin{abstract}
Let $M$ be a $G$-manifold and $\omega$ a $G$-invariant exact $m$-form on $M$. We indicate when these data allow us to constract a cocycle on a group $G$ with values in the trivial $G$-module $\mathbb{R}$ and when this cocycle is nontrivial.
\end{abstract}

\section{INTRODUCTION}

Let $M$ be a manifold, let $G$ be a group of diffeomorphisms of $M$, and let $\omega$ be a $G$-invariant exact $m$-form on $M$. In this paper we apply the construction of [5] to get from these data a cocycle on the group $G$ with values in the trivial $G$-module $\mathbb{R}$. We prove that this cocycle may be chosen differentiable (continuous) whenever $G$ is a subgroup of a Lie group (a topological group). Moreover, we prove that for a manifold $\mathbb{R}^{n} \times M$ with an exact form $\omega$ which is either of type $\omega_{0}+\omega_{M}$ or of type $\omega_{0} \wedge \omega_{M}$, where $\omega_{0}$ is a nonzero form on $\mathbb{R}^{n}$ with constant coefficients and $\omega_{M}$ is a form on $M$, and the group $\operatorname{Diff}\left(\mathbb{R}^{n} \times M, \omega\right)$ of diffeomorphisms of $\mathbb{R}^{n} \times M$ preserving the form $\omega$ the corresponding cocycle is nontrivial.

\section{A CONSTRUCtion of COHOMOLOGy Classes For A GROUP OF DIFFEOMORPHISMS}

Let $G$ be a group and let $A$ be a right $G$-module. Let $C^{p}(G, A)$ be the set of maps from $G^{p}$ to $A$ for $p>0$ and let $C^{0}(G, A)=A$. Define the differential $D: C^{p}(G, A) \rightarrow C^{p+1}(G, A)$ as follows, for $f \in C^{p}(G, A)$ and $g_{1}, \ldots, g_{p+1} \in G$ :

$$
\begin{aligned}
& (D f)\left(g_{1}, \ldots, g_{p+1}\right)=f\left(g_{2}, \ldots, g_{p+1}\right) \\
& \quad+\sum_{i=1}^{p}(-1)^{i} f\left(g_{1}, \ldots, g_{i} g_{i+1}, \ldots, g_{p+1}\right)+(-1)^{p+1} f\left(g_{1}, \ldots, g_{p}\right) g_{p+1} .
\end{aligned}
$$

Then $C^{*}(G, A)=\left(C^{p}(G, A), D\right)_{p \geq 0}$ is the standard complex of nonhomogeneous cochains of the group $G$ with values in the right $G$-module $A$ and its cohomology $H^{*}(G, A)=\left(H^{p}(G, A)_{p \geq 0}\right)$ is the cohomology of the group $G$ with values in $A$.

Date: October 4, 2018.

2000 Mathematics Subject Classification. Primary 58D05, 20J06, 22 E65.

Key words and phrases. group cohomology.

Supported by FWF Projekt P 17108-N04. 
Let $M$ be a smooth $n$-dimensional $G$-manifold, where $G$ is a group of diffeomorphisms of $M$. Denote by $\Omega(M)=\left(\Omega^{p}(M)\right)_{p=1, \ldots, n}$ the de Rham complex of differential forms on $M$ and consider the natural (right) action of the group $G$ on $\Omega(M)$ by pull backs. Denote by $\Omega(M)^{G}$ the subcomplex of $\Omega(M)$ consisting of $G$-invariant forms. Next we denote by $H_{q}(M)$ the $q$-dimensional real homology of $M$ and by $H^{q}(M)$ the $q$-dimensional real cohomology of $M$.

Let $\left.C^{*}(G, \Omega(M))=\left\{C^{p}\left(G, \Omega^{q}(M)\right), \delta^{\prime}\right)\right\}_{p, q \geq 0}$ be the standard complex of nonhomogeneous cochains of $G$ with values in the $G$-module $\Omega(M)$. We define the second differential $\delta^{\prime \prime}: C^{p}\left(G, \Omega^{q}(M)\right) \rightarrow C^{p}\left(G, \Omega^{q+1}(M)\right)$ by

$$
\left(\delta^{\prime \prime} c\right)\left(g_{1}, \ldots, g_{p}\right)=(-1)^{p} d c\left(g_{1}, \ldots, g_{p}\right),
$$

where $f \in C^{p}\left(G, \Omega^{q}(M)\right), g_{1}, \ldots, g_{p} \in G$, and where $d$ is exterior derivative. Since $\delta^{\prime} \delta^{\prime \prime}+\delta^{\prime \prime} \delta^{\prime}=0$, we have on $C^{*}(G, \Omega(M))$ the structure of double complex. Denote by $C^{* *}(G, \Omega(M))$ the cochain complex $C^{*}(G, \Omega(M))$ with respect to the total differential $\delta=\delta^{\prime}+\delta^{\prime \prime}$. We denote by $H(G, M, \Omega(M))$ the cohomology of the complex $C^{* *}(G, \Omega(M))$.

It is easily checked that the inclusion $\Omega(M)^{G} \subset C^{0}(G, \Omega(M))$ induces an injective homomorphism of complexes $\Omega(M)^{G} \rightarrow C^{* *}(G, \Omega(M))$ and thus also a homomorphism $H\left(\Omega(M)^{G}\right) \rightarrow H(G, M, \Omega(M))$ of cohomologies. We identify $\omega \in \Omega(M)^{G}$ with its image by the inclusion $\Omega(M)^{G} \subset C^{* *}(G, \Omega(M))$ and denote by $h(\omega)$ the cohomology class of $\omega$ in the complex $C^{* *}(G, \Omega(M))$ whenever the form $\omega$ is closed.

We shall use some standard facts on spectral sequences (see, for example, [1]). Consider the first filtration

$$
F_{1, p}(G, M, \Omega(M)):=\oplus_{q \geq p} C^{q}(G, \Omega(M))
$$

of the double complex $C^{*}(G, \Omega(M))$. By definition, $F_{1, p}(G, M, \Omega(M))$ is a subcomplex of the complex $C^{* *}(G, \Omega(M))$ and $F_{1,0}(G, M, \Omega(M))=C^{* *}(G, \Omega(M))$. Denote by $E_{1, r}=\left(E_{1, r}^{p, q}, d_{1, r}^{p q}\right)_{p, q \geq 0}$ for $r=0,1, \ldots, \infty$ the corresponding spectral sequence. Denote by $h_{p}$ the homomorphism of cohomologies $H\left(F_{1, p}(G, M, \Omega(M)) \rightarrow\right.$ $H(G, M, \Omega(M))$ induced by the inclusion $F_{1, p}(G, M, \Omega(M)) \subset C^{* *}(G, \Omega(M))$. Then $\left(\operatorname{Im} h_{p}\right)_{p \geq 0}$ is a filtration of the cohomology $H(G, M, \Omega(M))$ and

$$
E_{1, \infty}^{p, q}=h_{p}\left(H^{p+q}\left(F_{1, p}(G, M, \Omega(M))\right)\right) / h_{p+1}\left(H^{p+q}\left(F_{1, p+1}(G, M, \Omega(M))\right)\right) .
$$

For this spectral sequence we have $E_{1,2}^{p, q}=H^{p}\left(G, H^{q}(M)\right)$, where an action of the group $G$ on $H^{q}(M)$ is induced by its action on $\Omega(M)$. Moreover, there is a natural homomorphism $H^{p}\left(G, H^{0}(M)\right)=E_{1,2}^{p, 0} \rightarrow E_{\infty}^{p, 0}$.

Proposition 2.1. Let $\omega \in \Omega(M)^{G}$ be an exact $m$-form and let $p$ be the maximal integer such that $h(\omega) \in h_{p+1}\left(H^{m}\left(F_{1, p+1}(G, M, \Omega(M))\right)\right)$. Then the image of $h(\omega)$ under the natural homomorphism $\operatorname{Im} h_{p+1} \rightarrow \operatorname{Im} h_{p+1} / \operatorname{Im} h_{p+2}$ belongs to $E_{1, m-p+1}^{p+1, m-p-1}$. In particular, if $m=p+1$ and the manifold $M$ is connected, the above image of $h(\omega)$ is an $m$-dimensional cohomology class of the group $G$ with values in the trivial $G$-module $\mathbb{R}$. 
Proof. By assumption the image of $h(\omega)$ under the homomorphism

$$
\operatorname{Im} h_{p+1} \rightarrow \operatorname{Im} h_{p+1} / \operatorname{Im} h_{p+2}
$$

belongs to $E_{1, \infty}^{p+1, m-p-1}$. Since $d_{1, r}^{p, q}: E_{1, r}^{p, q} \rightarrow E_{1, r}^{p+r, q-r+1}$ vanishes whenever $r>q+1$, we have $E_{1, \infty}^{p+1, m-p-1}=E_{1, m-p+1}^{p+1, m-p-1}$. If $m=p+1$ and the manifold $M$ is connected we have $E_{1, \infty}^{p+1, m-p-1}=E_{2}^{m, 0}=H^{m}\left(G, H^{0}(M)\right)=H^{m}(G, \mathbb{R})$.

Theorem 2.2. Let $\omega$ be a $G$-invariant exact $m$-form on $M$ and let $H^{m-p}(M)=$ $\cdots=H^{m-1}(M)=0$ and $H^{m-p-1}(M) \neq 0$ for some $1 \leq p \leq m-1$. Then $h(\omega) \in \operatorname{Im} h_{p+1}$ and $h(\omega)$ defines a unique $(p+1)$-dimensional cohomology class $c(\omega)$ of the group $G$ with values in the natural $G$-module $H^{m-p-1}(M)$.

Proof. By assumption there is a form $\varphi_{0, m-1} \in C^{0}\left(G, \Omega^{m-1}(M)\right)$ such that $\omega=$ $-d \varphi_{0, m-1}=-\delta^{\prime \prime} \varphi_{0, m-1}$. Then we have $\omega+\delta \varphi_{0, m-1}=\delta^{\prime} \varphi_{0, m-1}$.

Since $H^{m-1}(M)=0$ and $\delta^{\prime \prime} \delta^{\prime} \varphi_{0, m-1}=-\delta^{\prime} \delta^{\prime \prime} \varphi_{0, m-1}=\delta^{\prime} \omega=0$, there is a cochain $\varphi_{1, m-2} \in C^{1}\left(G, \Omega^{m-2}(M)\right)$ such that $\delta^{\prime} \varphi_{0, m-1}=-\delta^{\prime \prime} \varphi_{1, m-2}$. Thus we have $\omega+\delta\left(\varphi_{0, m-1}+\varphi_{1, m-2}\right)=\delta^{\prime} \varphi_{1, m-2}$.

Using the conditions $H^{m-2}(M)=\cdots=H^{m-p}(M)=0$ and proceeding in the same way we get for $i=1, \ldots, p$ the cochains $\varphi_{i, m-i-1} \in C^{i}\left(G, \Omega^{m-i-1}(M)\right)$ such that

$$
\delta^{\prime} \varphi_{i-1, m-i}+\delta^{\prime \prime} \varphi_{i, m-i-1}=0
$$

and so

$$
\omega+\delta\left(\varphi_{0, m-1}+\cdots+\varphi_{p, m-p-1}\right)=\delta^{\prime} \varphi_{p, m-p-1} \in C^{p+1}\left(G, \Omega^{m-p-1}(M)\right) .
$$

Moreover, we have

$$
d \delta^{\prime} \varphi_{p, m-p-1}=-\delta^{\prime \prime} \delta^{\prime} \varphi_{p, m-p-1}=\delta^{\prime \prime} \delta^{\prime \prime} \varphi_{p-1, m-p}=0 .
$$

Consider $H^{m-p-1}(M)$ as a $G$-module with respect to the natural action of $G$ on $H^{m-p-1}(M)$. Then the cochain $\delta^{\prime} \varphi_{p, m-p-1}$ defines a cocycle on $G$ of degree $p+1$ with values in the $G$-module $H^{m-p-1}(M)$. We claim that the cohomology class of this cocycle depends only on the cohomology class of $\omega$ in the complex $\Omega(M)^{G}$.

If we replace the form $\omega$ by a form $\omega+d \omega_{1}$, where $\omega_{1} \in \Omega^{m-1}(M) \cap \Omega(M)^{G}$, one can replace the sequence $\varphi_{0, m-1}, \ldots, \varphi_{p, m-p-1}$ by the sequence $\varphi_{0, m-1}-$ $\omega_{1}, \varphi_{1, m-2}, \ldots, \varphi_{p, m-p-1}$ and obtain the same cochain $\varphi_{p, m-p-1}$ at the end.

Consider another sequence $\bar{\varphi}_{0, m-1}, \ldots, \bar{\varphi}_{p, m-p-1}(i=0, \ldots, p)$ such that $\omega=$ $-d \bar{\varphi}_{0, m-1}$ and $\delta^{\prime} \bar{\varphi}_{i-1, m-i}+\delta^{\prime \prime} \bar{\varphi}_{i, m-i-1}=0$ for $i=1, \ldots, p$. Since $H^{m-1}(M)=0$ we have

$$
\bar{\varphi}_{0, m-1}=\varphi_{0, m-1}+\delta^{\prime \prime} \psi_{0, m-2},
$$

where $\psi_{0, m-2} \in C^{0}\left(G, \Omega^{m-2}(M)\right)$. If $p=1$ we have $\delta^{\prime} \bar{\varphi}_{0, m-1}=\delta^{\prime} \varphi_{0, m-1}-$ $\delta^{\prime \prime} \delta^{\prime} \psi_{0, m-2}$ and we are done. If $p>1$ we have

$$
\delta^{\prime} \bar{\varphi}_{0, m-1}=\delta^{\prime} \varphi_{0, m-1}-\delta^{\prime \prime} \delta^{\prime} \psi_{0, m-2}=-\delta^{\prime \prime}\left(\varphi_{1, m-2}+\delta^{\prime} \psi_{0, m-2}\right)=-\delta^{\prime \prime} \bar{\varphi}_{1, m-2} .
$$


Since $H^{m-2}(M)=0$ there is a cochain $\psi_{1, m-3} \in C^{1}\left(G, \Omega^{m-3}(M)\right)$ such that $\bar{\varphi}_{1, m-2}=\varphi_{1, p-2}+\delta^{\prime} \psi_{0, m-2}+\delta^{\prime \prime} \psi_{1, m-3}$. For $i=2, \ldots, p-1$ proceeding in the same way we get the cochains $\psi_{i, m-i-2} \in C^{i}\left(G, \Omega^{m-i-2}(M)\right)$ such that

$$
\bar{\varphi}_{i, m-i-1}=\varphi_{i, m-i-1}+\delta^{\prime} \psi_{i-1, m-i-1}+\delta^{\prime \prime} \psi_{i, m-i-2} \text {. }
$$

In particular, we have

$$
\bar{\varphi}_{p, m-p-1}=\varphi_{p, m-p-1}+\delta^{\prime} \psi_{p-1, m-p-1}+\delta^{\prime \prime} \psi_{p, m-p-2}
$$

and $\delta^{\prime} \bar{\varphi}_{p, m-p-1}=\delta^{\prime} \varphi_{p, m-p-1}-\delta^{\prime \prime} \psi_{p, m-p-2}$. Thus the cochains $\delta^{\prime} \bar{\varphi}_{p, m-p-1}$ and $\delta^{\prime} \varphi_{p, m-p-1}$ define the same cohomology class of $H^{p+1}\left(G, H^{m-p-1}(M)\right)$.

Suppose that the conditions of theorem 2.2 are satisfied for an exact $m$-form $\omega \in \Omega(M)^{G}$. Let $\alpha$ be a singular smooth cycle of $M$ of dimension $m-p-1$ whose homology class $a$ is invariant under the natural action of the group $G$ on $H_{m-p-1}(M)$. Put

$$
c_{a}(\omega)\left(g_{1}, \ldots, g_{p+1}\right)=\int_{\alpha}\left(\delta^{\prime} \varphi_{p, m-p-1}\right)\left(g_{1}, \ldots, g_{p+1}\right) .
$$

By definition, $c_{a}(\omega)$ is a $(p+1)$-cocycle on the group $G$ with values in the trivial $G$-module $\mathbb{R}$ which is independent of a choice of the cycle $\alpha$ in the homology class $a$.

Let $p=0$. Evidently, the cocycle $c_{a}(\omega)$ is nontrivial if and only if it does not vanish. From now on we assume $p>0$.

Remark 2.3. Let the assumptions of theorem 2.2 be satisfied for an exact $m$-form $\omega \in \Omega(M)^{G}$. If either the manifold is connected and $m=p+1$, or $G$ is a connected topological group, the action of $G$ on the $H_{m-p-1}(M)$ is trivial. If the homology class $a$ of the cycle $\alpha$ is not invariant under the action of $G$, consider the vector subspace $H_{a}$ of $H_{m-p-1}(M)$ generated by the orbit of $a$. Then (3) defines a $(p+1)-$ cocycle on the group $G$ with values in the $G$-module $H_{a}$.

Consider the partial case when $M=G$ is a connected Lie group and the group $G$ acts on $M$ by left translations. It is clear that the complex $\Omega(G)^{G}$ is isomorphic to the complex $C^{*}(\mathfrak{g}, \mathbb{R})$ of standard cochains of the Lie algebra $\mathfrak{g}$ of the group $G$ with values in the trivial $\mathfrak{g}$-module $\mathbb{R}$. Consider the second filtration

$$
F_{2, p} C^{* *}(G, G, \Omega(G)):=\oplus_{q \geq p} C^{*}\left(G, \Omega^{q}(G)\right)
$$

of the double complex $C^{* *}(G, \Omega(G))$ and the corresponding spectral sequence $E_{2, r}=$ $\left(E_{2, r}^{p, q}, d_{r}^{p, q}\right)_{p, q \geq 0}$ for $r=0,1, \ldots, \infty$. It is easily seen that $E_{2,1}^{p, q}=H^{p}\left(G, \Omega^{q}(G)\right)$.

Lemma 2.4. The inclusion $\Omega(M)^{G} \subset C^{* *}(G, G, \Omega(G))$ induces an isomorphism of cohomologies.

Proof. We prove that for each $q \geq 0$ we have

$$
H^{p}\left(G, \Omega^{q}(G)\right)=0 \text { for } p>0 \text { and } H^{0}\left(G, \Omega^{q}(G)\right)=\Omega^{q}(G)^{G} .
$$


First we consider the case when $q=0$. We use the standard operator $B$ : $C^{p}\left(G, \Omega^{0}(G)\right) \rightarrow C^{p-1}\left(G, \Omega^{0}(G)\right)$ defined as follows. For $p>0$, put

$$
(B c)\left(g_{1}, \ldots, g_{p-1}\right)(g)=(-1)^{p} c\left(g_{1}, \ldots, g_{p-1}, g\right)(e),
$$

where $c \in C^{p}\left(G, \Omega^{0}(G)\right), g, g_{1}, \ldots, g_{p-1} \in G$, and $e$ is the identity element of $G$. For $c \in C^{0}\left(G, \Omega^{0}(G)\right)$, put $B c=0$. It is easy to check that $B$ is a homotopy operator between the identity isomorphism of $C^{*}\left(G, \Omega^{0}(G)\right)$ and the map of $C^{*}\left(G, \Omega^{0}(G)\right)$ into itself which vanishes on $C^{p}\left(G, \Omega^{0}(G)\right)$ for $p>0$ and takes $\left.c \in C^{0}\left(G, \Omega^{0}\right)\right)$ to $c(e)$. This proves our statement for $p=0$.

To prove our statement for $p>0$ we note that $\Omega^{q}(G)=\Omega^{q}(G)^{G} \otimes \Omega^{0}(G)$, where $\Omega^{p}(G)^{G}$ is the space of left invariant $q$-forms on $G$. Since $G$ acts trivially on $\Omega^{p}(G)^{G}$, its action on $\Omega^{q}(G)$ is induced by its action on $\Omega^{0}(G)$. Then we have $H^{p}\left(G, \Omega^{q}(G)\right)=0$ for $p>0$ and $H^{0}\left(G, \Omega^{q}(G)\right)=\Omega^{q}(G)^{G}$.

The above statement implies that $E_{2,1}^{p, q}=0$ when $p>0$ and $E_{2,1}^{0, q}=\Omega^{q}(G)^{G}$. Then $E_{2,1}=\Omega(G)^{G}$ and evidently the differential $d_{2,1}^{0, q}$ equals the exterior derivative $d$ on $\Omega(G)^{G}$ up to sign. Therefore we have $E_{2,2}^{p, q}=0$ when $p>0$ and $E_{2,2}^{0, q}=$ $H^{q}\left(\Omega(G)^{G}\right)$. Thus implies that $E_{2, \infty}^{p, q}=E_{2,2}^{p, q}$ and therefore the inclusion $\Omega(G)^{G} \subset$ $C^{* *}(G, \Omega(G))$ induces an isomorphism of cohomologies.

Proposition 2.5. Let $\omega \in \Omega(G)^{G}$ be an exact $m$-form whose cohomology class in the complex $\Omega(G)^{G}$ is nontrivial and let

$$
\varphi_{i, m-i-1} \in C^{i}\left(G, \Omega^{m-i-1}(G)\right) \quad(i=0, \ldots, m-1)
$$

be a sequence of cochains such that $\delta\left(\omega+\varphi_{0, m-1}+\cdots+\varphi_{m-1,0}\right)=\delta^{\prime} \varphi_{m-1,0}$. Then, for a point $x \in G$, a cocycle $\delta^{\prime} \varphi_{m-1,0}(g)(x)$ of the complex $C^{*}(G, \mathbb{R})$ is nontrivial.

Proof. By lemma 2.4 the cohomology class of $\omega$ in the complex $C^{* *}(G, \Omega(G))$ is nontrivial and then by assumption $\omega$ defines a nontrivial element of $E_{2, \infty}^{m, 0}$. Since

$$
H^{m}(G, \mathbb{R})=E_{2,2}^{m, 0}=E_{2, \infty}^{m, 0},
$$

the cocycle $\delta^{\prime} \varphi_{m-1,0}(g)(x)$ of the complex $C^{*}(G, \mathbb{R})$ is nontrivial.

\section{The MAP $f_{\gamma}$ AND ITS PROPERTIES}

Let $G$ be a finite dimensional Lie group. For $X \in T_{e}(M)$ denote by $X^{r}$ the right invariant vector field on $G$ such that $X^{r}(e)=X$ and by $\tilde{X}$ the fundamental vector field on $M$ corresponding to $X$ for the action of $G$ on $M$. We denote the action by $\varphi: G \times M \rightarrow M$ and write $g x=\varphi(g, x)=\varphi^{x}(g)=\varphi_{g}(x)$. By definition, $T\left(\varphi^{x}\right) X^{r}(g)=\tilde{X}(g x)$ and for each $g \in G$ we have $\varphi^{x} \circ \mathrm{L}_{g}=\varphi_{g} \circ \varphi^{x}$, where $\mathrm{L}_{g}$ is left translation on $G$.

Let $\gamma$ be a singular smooth cycle of dimension $q$ on $M$. Define a map $f_{\gamma}$ : $\Omega(M) \rightarrow \Omega(G)$ as follows. Let $\omega \in \Omega(M)$. If $\operatorname{deg} \omega<q$ put $f_{\gamma}(\omega)=0$. If 
$\operatorname{deg} \omega=p+q$ with $p \geq 0$ put

$$
f_{\gamma}(\omega)\left(X_{1}^{r}, \ldots, X_{p}^{r}\right)(g)=\int_{\gamma} \varphi_{g}^{*}\left(i\left(\tilde{X}_{p}\right) \ldots i\left(\tilde{X}_{1}\right) \omega\right)=\int_{g \gamma} i\left(\tilde{X}_{p}\right) \ldots i\left(\tilde{X}_{1}\right) \omega
$$

where $X_{1}, \ldots, X_{p} \in T_{e}(G)$ and $g \in G$. Clearly $\omega \rightarrow f_{\gamma}(\omega)$ is a linear map from $\Omega(M)$ to $\Omega(G)$ decreasing degrees to $q$.

Consider the action of the group $G$ on itself by left translations.

Lemma 3.1. The map $f_{\gamma}$ is G-equivariant.

Proof. It suffices to consider the case when $\operatorname{deg} \omega \geq q$. Let $\omega \in \Omega^{p+q}(M), X \in$ $T_{e}(G)$, and $g, \tilde{g} \in G$. It is easy to check that

$$
\begin{gathered}
T\left(\mathrm{~L}_{\tilde{g}}\right) \circ X^{r}=(\operatorname{ad} \tilde{g}(X))^{r} \circ L_{\tilde{g}}: G \rightarrow T G, \\
T\left(\varphi_{\tilde{g}}\right) \circ \tilde{X}=\widehat{\operatorname{ad} \tilde{g}(X) \circ \varphi_{\tilde{g}}: M \rightarrow T M}
\end{gathered}
$$

From this we get

$$
\begin{aligned}
& \left.L_{\tilde{g}}^{*} f_{\gamma}(\omega)\right)\left(X_{1}^{r}, \ldots, X_{p}^{r}\right)(g)=\int_{\tilde{g} g \gamma} i\left(T \varphi_{\tilde{g}} \cdot \tilde{X}_{p}\right) \ldots i\left(T \varphi_{\tilde{g}} \cdot \tilde{X}_{1}\right) \omega \\
& \left.\quad=\int_{g(\gamma)} \varphi_{g}^{*}\left(i\left(T \varphi_{\tilde{g}} \tilde{X}_{p}\right) \ldots i\left(T \varphi_{\tilde{g}} \cdot \tilde{X}_{1}\right)\right) \omega\right)=\int_{g \gamma} i\left(\tilde{X}_{p}\right) \ldots i\left(\tilde{X}_{1}\right) \varphi_{g}^{*} \omega=f_{\gamma}\left(\varphi_{g}^{*} \omega\right) .
\end{aligned}
$$

Lemma 3.2. $d_{G} \circ f_{\gamma}=f_{\gamma} \circ d$, where $d_{G}$ is the exterior derivative in $\Omega(G)$.

Proof. Let $\omega \in \Omega^{m}(M)$. If $m<q$, by definition we have $d_{G} \circ f_{\gamma}(\omega)=f_{\gamma} \circ d(\omega)=0$.

Let $\omega \in \Omega^{p+q}(M)$, where $p \geq 0$, and $X_{1}, \ldots, X_{p} \in T_{e}(G)$. Then we have

$$
\begin{gathered}
\left(d_{G} f_{\gamma}(\omega)\right)\left(X_{1}^{r}, \ldots, X_{p}^{r}\right)(g)=\sum_{i=1}^{p}(-1)^{i-1} X_{i}^{r}(g) f_{\gamma}(\omega)\left(X_{1}^{r}, \ldots, \widehat{X}_{i}^{r}, \ldots, X_{p}^{r}\right) \\
+\sum_{i<j}(-1)^{i+j} f_{\gamma}(\omega)\left(\left[X_{i}^{r}, X_{j}^{r}\right], X_{1}^{r}, \ldots, \widehat{X}_{i}^{r}, \ldots, \widehat{X}_{j}^{r} \ldots, \ldots, X_{p}^{r}\right)(g) \\
=\sum_{i=1}^{p}(-1)^{i-1} X_{i}^{r}(g) \int_{\gamma} g^{*}\left(i\left(\tilde{X}_{p}\right) \ldots \widehat{i\left(\tilde{X}_{i}\right)} \ldots i\left(\tilde{X}_{1}\right) \omega\right) \\
+\sum_{i<j}(-1)^{i+j} \int_{\gamma} g^{*}\left(i\left(\tilde{X}_{p}\right) \ldots \widehat{i\left(\tilde{X}_{j}\right)} \ldots \widehat{i\left(\tilde{X}_{i}\right)} \ldots i\left(\tilde{X}_{1}\right) i\left(\left[\tilde{X}_{i}, \tilde{X}_{j}\right]\right) \omega\right) \\
=\sum_{i=1}^{p}(-1)^{i-1} \int_{\gamma} g^{*}\left(\mathrm{~L}_{\tilde{X}_{i}}\left(i\left(\tilde{X}_{p}\right) \ldots \widehat{i\left(\tilde{X}_{i}\right)} \ldots i\left(\tilde{X}_{1}\right) \omega\right)\right. \\
+\sum_{i<j}(-1)^{i+j} \int_{\gamma} g^{*}\left(i\left(\tilde{X}_{p}\right) \ldots \widehat{i\left(\tilde{X}_{j}\right)} \ldots \widehat{i\left(\tilde{X}_{i}\right)} \ldots i\left(\tilde{X}_{1}\right) i\left(\left[\tilde{X}_{i}, \tilde{X}_{j}\right]\right) \omega\right)
\end{gathered}
$$


where $\mathrm{L}_{X}$ denote the Lie derivative with respect to a vector field $X$ and, as usual, $\widehat{i(\tilde{X})}$ means that the term $i(\tilde{X})$ is omitted.

Using the formula $\left[\mathrm{L}_{X}, i(Y)\right]=i([X, Y])$ it is easy to check by induction over $p$ that for any manifold $M$ and vector fields $X_{1}, \ldots, X_{p}$ on $M$ the following formula is true.

$$
\begin{aligned}
& \sum_{i=1}^{p}(-1)^{i-1} \mathrm{~L}_{X_{i}} i\left(X_{p}\right) \ldots \widehat{i\left(X_{i}\right)} \ldots i\left(X_{1}\right) \\
&+\sum_{i<j}(-1)^{i+j} i\left(X_{p}\right) \ldots \widehat{i\left(X_{j}\right)} \ldots \widehat{i\left(X_{i}\right)} \ldots i\left(X_{1}\right) i\left(\left[X_{i}, X_{j}\right]\right. \\
& \quad=i\left(X_{q}\right) \ldots i\left(X_{1}\right) d+(-1)^{p-1} d i\left(X_{q}\right) \ldots i\left(X_{1}\right) .
\end{aligned}
$$

Applying this formula in (7) we get

$$
\left(d_{G} f_{\gamma}\left(\omega_{1}\right)\right)\left(X_{1}^{r}, \ldots, X_{p}^{r}\right)(g)=\int_{\gamma} g^{*} i\left(\tilde{X}_{p}\right) \ldots i\left(\tilde{X}_{1}\right) d \omega=f_{\gamma}(d \omega)\left(X_{1}^{r}, \ldots, X_{p}^{r}\right)(g) .
$$

Consider the double complex $\left(C^{*}(G, \Omega(G)), \delta_{G}^{\prime}, \delta_{G}^{\prime \prime}\right)$ for the action of the group $G$ on $G$ by left translations. Define the map $F_{\gamma}:\left(C^{* *}(M, \Omega(M))\left(C^{* *}(G, \Omega(G))\right.\right.$ as follows: for a cochain $c \in C^{p}\left(G, \Omega^{q}(M)\right)$ put $F_{\gamma}(c)=f_{\gamma} \circ c$.

Lemma 3.3. $\delta_{G}^{\prime} \circ F_{\gamma}=F_{\gamma} \circ \delta^{\prime}$.

Proof. Let $c \in C^{s}\left(G, \Omega^{p+q}(M)\right.$ and $g, g_{1}, \ldots, g_{s+1} \in G$. By definition we have

(8) $\left.\left(\delta_{G}^{\prime} \circ F_{\gamma}\right)(c)\right)\left(g_{1}, \ldots, g_{s+1}\right)(g)=F_{\gamma}(c)\left(g_{2}, \ldots, g_{s+1}\right)(g)$

$+\sum_{i=1}^{s}(-1)^{i} F_{\gamma}(c)\left(g_{1}, \ldots, g_{i} g_{i+1}, \ldots, g_{s+1}\right)(g)+(-1)^{s+1} \mathrm{~L}_{g_{s+1}}^{*} F_{\gamma}(c)\left(g_{1}, \ldots, g_{s}\right)(g)$.

For $X_{1}, \ldots, X_{p} \in T_{e}(G)$ and $g \in G$ by (15) and (6) we get

$$
\begin{aligned}
& \mathrm{L}_{g_{s+1}}^{*} F_{\gamma}(c)\left(g_{1}, \ldots, g_{s}\right)\left(X_{1}^{r}, \ldots, X_{p}^{s}\right)(g) \\
&\left.=\int_{\gamma}\left(g_{s+1} g\right)^{*}\left(i\left(\operatorname{ad} \widetilde{g_{s+1}\left(X_{p}\right.}\right)\right) \ldots i\left(\widetilde{\operatorname{ad}} \widetilde{g_{s+1}\left(X_{1}\right)}\right) c\left(g_{1}, \ldots g_{s}\right)\right) \\
&=\int_{\gamma} g^{*}\left(i\left(\tilde{X}_{p}\right) \ldots i\left(\tilde{X}_{1}\right) c\left(g_{1}, \ldots, g_{s}\right)\right)=F_{\gamma}\left(g_{p+1}^{*} c\left(g_{1}, \ldots, g_{s}\right)\right)\left(X_{1}^{r}, \ldots, X_{p}^{s}\right)(g) .
\end{aligned}
$$

Replacing the last summand in (8) by formula (9) we get

$$
\left(\delta_{G}^{\prime} \circ F_{\gamma}\right)(c)\left(g_{1}, \ldots, g_{s+1}\right)(g)=\left(F_{\gamma} \circ \delta^{\prime}\right)(c)\left(g_{1}, \ldots, g_{s+1}\right)(g) .
$$

Lemmas 3.113.2 and (8) imply the following 
Theorem 3.4. The map $F_{\gamma}: C^{*}(G, \Omega(M)) \rightarrow C^{*}(G, \Omega(G))$ is a homomorphism of double complexes decreasing the second degree to $q$.

Suppose that the conditions of theorem 2.2 for an exact $m$-form $\omega$ are satisfied. Let $\alpha$ be a singular smooth cycle of $M$ of dimension $m-p-1$ whose homology class $a$ is invariant under the natural action of the group $G$ on $H_{m-p-1}(M)$. Consider the sequence of cochains $\varphi_{i, m-i-1}(i=0, \ldots, p)$ constructed in the proof of theorem 2.2 By theorem 3.4 $F_{\alpha} \circ \omega$ is a left invariant $(p+1)$-form on $G$, i.e., a $(p+1)$-cocycle of the complex $C^{*}(\mathfrak{g}, \mathbb{R})$. Moreover, we have

$$
\delta_{G}^{\prime} F_{\alpha} \circ \varphi_{i-1, m-i}+\delta_{G}^{\prime \prime} F_{\alpha} \circ \varphi_{i, m-i-1}=0 \quad(i=1, \ldots, p) .
$$

Since $d_{G}\left(\delta_{G}^{\prime} \circ F_{\alpha} \circ \varphi_{p, m-p-1}\right)=0$, for any $g \in G$ we have

$$
c_{a}(\omega)=\int_{\gamma} \delta^{\prime} \varphi_{p, m-p-1}=\left(F_{\alpha} \circ \delta^{\prime} \circ \varphi_{p, m-p-1}\right)(e)=\left(\delta^{\prime} \circ F_{\alpha} \circ \varphi_{p, m-p-1}\right)(e) .
$$

Consider the complex $\left(C^{*}(G, \mathbb{R}), D\right)$ of nonhomogeneous cochains on the group $G$ with values in the trivial $G$-module $\mathbb{R}$. Define a cochain $b \in C^{p}(G, \mathbb{R})$ as follows:

$$
b\left(g_{1}, \ldots, g_{p}\right)=\int_{\alpha} \varphi_{p, m-p-1}\left(g_{1}, \ldots, g_{p}\right) .
$$

By the definitions of the cocycle $c_{a}(\omega)$ and the map $f_{\gamma}$ and by (11) we have

$$
\begin{aligned}
c_{a}\left(g_{1}, \ldots, g_{p}, g\right)=(-1)^{p+1} & \left(f_{\alpha} \circ \varphi_{p, m-p-1}\left(g_{1}, \ldots, g_{p}\right)(g)-b\left(g_{1}, \ldots, g_{p}\right)\right) \\
+ & (D b)\left(g_{1}, \ldots, g_{p}, g\right) .
\end{aligned}
$$

Proposition 3.5. Let the cycle $\gamma$ be $G$-invariant. Then the cocycle $c_{a}(\omega)$ is trivial.

Proof. By assumption we have

$$
\left(F_{\alpha} \circ \varphi_{p, m-p-1}\right)\left(g_{1}, \ldots, g_{p}\right)(g)=\int_{g \alpha} \varphi_{p, m-p-1}\left(g_{1}, \ldots, g_{p}\right)=b\left(g_{1}, \ldots, g_{p}\right)
$$

Then by (10) we have $c_{a}(\omega)=D b$.

Denote by $H$ the subgroup of $G$ consisting of all elements $g \in G$ preserving the cycle $\gamma$. Consider the natural action of the group $G$ on the homogeneous space $G / H$. The projection $p_{H}: G \rightarrow G / H$ induces a homomorphism $\tilde{p}_{H}: C^{*}(G, \Omega(G / H)) \rightarrow$ $C^{*}(g, \Omega(G))$ of double complexes.

Proposition 3.6. There is a unique homomorphism of double complexes $F_{\gamma, H}$ : $C^{*}(G, \Omega(M)) \rightarrow C^{*}(G, \Omega(G / H))$ such that $F_{\gamma}=\tilde{p}_{H} \circ F_{\gamma, H}$.

Proof. Note that formula (4) implies that the form $f_{\gamma}(\omega)$ is $H$-invariant. Moreover, by assumption for each $X \in T_{e}(H)$ the fundamental vector field $\tilde{X}$ preserves the cycle $\gamma$. Thus the form $i(X) \omega$ vanishes on the cycle $\gamma$. This implies that $f_{\gamma}(\omega)\left(X_{1}^{r}, \ldots, X_{p}^{r}\right)=0$ whenever one of the vectors $X_{1}, \ldots, X_{p} \in T_{e}(G)$ belongs to $T_{e}(H)$. Thus the form $f_{\gamma}(\omega)$ lies in the image of the map $p^{*}: \Omega(G / H) \rightarrow \Omega(G)$. 

$c_{a}(\omega)$.

We point out the following sufficient condition of nontriviality of the cocycle

Theorem 3.7. Let $\omega \in \Omega(M)^{G}$ be an exact $m$-form such that the conditions of theorem 2.2 are satisfied and let $\alpha$ be a singular smooth $(m-p-1)$-cycle on $M$ whose homology class a is G-invariant. If the cohomology class of the closed left invariant form $f_{\alpha} \circ \omega$ in the complex $\Omega(G)^{G}$ is nontrivial, the cocycle $c_{a}(\omega)$ of the complex $C^{*}(G, \mathbb{R})$ is nontrivial as well.

Proof. It is easy to check that the form $f_{a} \circ \omega$ satisfies the conditions of proposition 2.5 and the cocycle $c_{a}(\omega)$ equals the cocycle $\delta_{G}^{\prime} \circ f_{\alpha} \circ \varphi_{p, m-p-1}(e)$. Thus the cocycle $c_{a}(\omega)$ is nontrivial.

Let $H$ be a subgroup $G$ preserving the cycle $\gamma$. By proposition [3.6 the condition of theorem [3.7 can be satisfied only if $\operatorname{dim} G / H \geq m$.

\section{Continuous and Differentiable Cocycles}

Let $G$ be a topological group (or a Lie group which may be infinite-dimensional), $E$ a Frechét space, and $\rho: G \rightarrow \operatorname{GL}(E)$ a representation of $G$ in $E$. A cochain $f \in C^{p}(G, E)$ is continuous (differentiable) if it is a continuous (differentiable of class $\left.C^{\infty}\right)$ map from $G^{p}$ to $E$. Let $C_{\mathrm{c}}^{p}(G, E)$ and $C_{\mathrm{diff}}^{p}(G, E)$ denote the subspaces of continuous and differentiable cochains of the space $C^{p}(G, E)$, respectively. Denote by $H_{\mathrm{c}}^{*}(G, E)$ and $H_{\mathrm{diff}}^{*}(G, E)$ the cohomology of the complex $C_{\mathrm{c}}^{*}(G, E)=$ $\left(C_{\mathrm{c}}^{p}(G, E), \delta^{\prime}\right)_{p \geq 0}$ and of $C_{\mathrm{c}}^{*}(G, E)=\left(C_{\mathrm{diff}}^{p}(G, E), \delta^{\prime}\right)_{p \geq 0}$, respectively. It is known (see [2] and [3]) that the inclusion $C_{\mathrm{diff}}^{*}(G, E) \subset C_{\mathrm{c}}^{*}(G, E)$ induces an isomorphism $H_{\mathrm{c}}^{*}(G, E)=H_{\mathrm{diff}}^{*}(G, E)$ whenever $G$ is a finite dimensional Lie group.

Later we apply these notions to $\Omega(M)$ as a topological vector space with the $C^{\infty}$ topology. Evidently both $C_{\mathrm{c}}^{* *}(G, \Omega(M))=\left(C_{\mathrm{c}}^{p}\left(G, \Omega^{q}(M)\right)\right)$ and $C_{\mathrm{diff}}^{* *}(G, \Omega(M))=$ $\left(C_{\text {diff }}^{p}\left(G, \Omega^{q}(M)\right)\right)$ are subcomplexes of $C^{* *}(G, \Omega(M))$.

Let the conditions of theorem 2.2 be satisfied for an exact $m$-form $\omega \in \Omega(M)^{G}$. Assume that $G$ is a topological group or a Lie group. We investigate whether we can construct a sequence $\varphi_{i, m-i-1}$ for $i=1, \ldots, p$ as above which consists of continuous or differentiable cochains. For such a sequence $c_{a}(\omega)$ is a continuous or differentiable cocycle.

Theorem 4.1. Let $M$ be a connected manifold with a countable base. Then for each $p>0$ we have the following decomposition in the category of topological vector spaces

$$
\Omega^{p}(M)=d \Omega^{p-1}(M) \oplus H^{p}(M) \oplus \Omega^{p}(M) / Z^{p}(M),
$$

where $Z^{p}(M)$ is the space of closed p-forms. If $H^{p}(M)=0, d \Omega^{p-1}(M)=Z^{p}(M)$ and $\Omega^{p}(M) / Z^{p}(M)$ are Frechét spaces.

Proof. For compact $M$ the statement follows from the Hodge decomposition for the identity operator 1 on $\Omega^{p}(M) 1=d \circ \delta \circ G \oplus H^{p}(M) \oplus \delta \circ d \circ G$ (see, for example, [9]). 
For noncompact $M$ the statement follows from Palamodov's theorem (see [8], Proposition 5.4).

Corollary 4.2. Let the conditions of theorem 2.2 be satisfied for an exact $m$-form $\omega \in \Omega(M)^{G}$ and let $G$ be a topological group (a Lie group). Then one can construct a sequence $\varphi_{i, m-i-1}$ for $i=1, \ldots, p$ consisting of continuous (differentiable) cochains and thus for a singular smooth $(m-p-1)$-dimensional cycle $\alpha$ whose homology class a is $G$-invariant the corresponding cocycle $c_{a}(\omega)$ is continuous (differentiable).

Proof. The sequence $\varphi_{i, m-i-1}(i=1, \ldots, p)$ is constructed successively by means of the equation $\delta^{\prime} \varphi_{i-1, m-i}+(-1)^{i} d \varphi_{i, m-i-1}=0$. By theorem 4.1 for each of the above cases this equation has a continuous (differentiable) solution $\varphi_{i, m-i-1}=$ $L_{m-i} \circ \delta^{\prime} \varphi_{i_{1}, m-i}$ whenever the cochain $\varphi_{i_{1}, m-i}$ is continuous (differentiable).

\section{Conditions of triviality of A Differentiable Cocycle $c_{a}(\omega)$}

In this section we study the conditions of triviality of the cocycle $c_{a}(\omega)$ in the complex $C_{\text {diff }}^{*}(G, \mathbb{R})$ whenever $G$ is a Lie group and for the exact $m$-form $\omega$ we choose a sequence of cochains $\varphi_{i, m-i-1}(i=1, \ldots, p)$ consisting of differentiable cochains.

Theorem 5.1. Let $M$ be a $G$-manifold, where $G$ is a Lie group preserving an exact $m$-form $\omega$, let the conditions of theorem 2.2 be satisfied, and for $i=1, \ldots, p$ let $\varphi_{i, m-i-1} \in C_{\mathrm{diff}}^{i}\left(G, \Omega^{m-i-1}(M)\right)$. Then, if the cocycle $c_{a}(\omega)$ is trivial, there is a cochain $f \in C_{\mathrm{diff}}^{p-1}\left(G, \Omega^{0}(G)\right)$ such that $\delta_{G}^{\prime}\left(f_{\gamma} \circ \varphi_{p-1, m-p}-d_{G} f\right)=0$. If the group $G$ is connected, this condition implies the triviality of the cocycle $c_{a}(\omega)$.

Proof. Let the cocycle $c_{a}(\omega)$ be trivial. By (10) there is a cochain $\bar{f} \in C_{\text {diff }}^{p}(G, \mathbb{R})$ such that for any $g, g_{1}, \ldots, g_{p} \in G$ we have

$$
(-1)^{p+1}\left(f_{a} \circ \varphi_{p, m-p-1}\left(g_{1}, \ldots, g_{p}\right)(g)-b\left(g_{1}, \ldots, g_{p}\right)\right)=D \bar{f}\left(g_{1}, \ldots, g_{p}, g\right) .
$$

Define a cochain $f \in C_{\mathrm{diff}}^{p-1}\left(G, \Omega^{0}(G)\right)$ as follows

$$
f\left(g_{1}, \ldots, g_{p-1}\right)(g)=\bar{f}\left(g_{1}, \ldots, g_{p-1}, g\right) .
$$

By lemma 3.2 we have

$$
\begin{aligned}
& d_{G}\left((-1)^{p+1}\left(\left(f_{a} \circ \varphi_{p, m-p-1}\right)\left(g_{1}, \ldots, g_{p}\right)(g)-b\left(g_{1}, \ldots, g_{p}\right)\right)\right. \\
&=(-1)^{p+1}\left(f_{\alpha} \circ d \varphi_{p, m-p-1}\right)\left(g_{1}, \ldots, g_{p}\right)(g) \\
& \quad=\delta_{G}^{\prime}\left(f_{\alpha} \circ \varphi_{p-1, m-p}\right)\left(g_{1}, \ldots, g_{p}\right)(g) .
\end{aligned}
$$

On the other hand, it is easy to check that

$$
D \bar{f}\left(g_{1}, \ldots, g_{p}, g\right)=\left(\delta_{G}^{\prime} f\right)\left(g_{1}, \ldots, g_{p}\right)(g)+(-1)^{p+1} \bar{f}\left(g_{1}, \ldots, g_{p}\right) .
$$


By (12) and (13), equation (11) implies

$$
\begin{aligned}
\left.\delta_{G}^{\prime}\left(f_{\alpha} \circ \varphi_{p-1, m-p}\right)\left(g_{1}, \ldots, g_{p}\right)(g)-\left(d_{G} \delta_{G}^{\prime} f\right)\left(g_{1}, \ldots, g_{p}\right)(g)\right) \\
\left.=\delta_{G}^{\prime}\left(\left(f_{\alpha} \circ \varphi_{p-1, m-p}-d_{G} f\right)\right)\left(g_{1}, \ldots, g_{p}\right)(g)\right)=0 .
\end{aligned}
$$

Now suppose that the condition of the theorem is satisfied. We may assume that for any $g_{1}, \ldots, g_{p} \in G$ we have $f\left(g_{1}, \ldots, g_{p}\right)(e)=0$. The above condition is equivalent to the following one

$$
\delta_{G}^{\prime \prime}\left((-1)^{p+1} f_{\alpha} \circ \varphi_{p, m-p-1}-\delta_{G}^{\prime} f\right)\left(g_{1}, \ldots, g_{p}\right)(g)=0 .
$$

Since the group $G$ is connected and $f\left(g_{1}, \ldots, g_{p-1}\right)(e)=0$ we have

$$
\begin{aligned}
\left((-1)^{p+1} f_{\alpha} \circ \varphi_{p, m-p-1}-\delta_{G}^{\prime} f\right)\left(g_{1}, \ldots, g_{p}\right)(g) & \\
=\left((-1)^{p+1} f_{\alpha} \circ \varphi_{p, m-p-1}-\delta_{G}^{\prime} f\right) & \left(g_{1}, \ldots, g_{p}\right)(e) \\
= & (-1)^{p+1}(b-\bar{f})\left(g_{1}, \ldots, g_{p}\right) .
\end{aligned}
$$

Using (10) and (13) we get $\left(c_{a}(\omega)-D \bar{f}\right)\left(g_{1}, \ldots, g_{p}, g\right)=0$. This concludes the proof.

Corollary 5.2. Let the conditions of theorem 5.1 be satisfied for $m=2$ and $p=1$. Then, if the 2-cocycle $c_{a}(\omega)$ is trivial, there is a smooth function $f$ on $G$ such that the 1-form $f_{\alpha} \circ\left(\varphi_{0,1}\right)-d_{G} f$ on $G$ is left invariant. In particular, the cohomology class of the form $f_{\alpha} \circ \omega$ in the complex $\Omega(G)^{G}$ is trivial. If the group $G$ is connected, the above condition implies the triviality of the cocycle $c_{a}(\omega)$.

Example 5.3. Consider the abelian group $G=\mathbb{R}^{n}$ acting on itself by translations. Evidently an $m$-form

$$
\omega(x)=\sum_{i_{1}<\cdots<i_{m}} \omega_{i_{1} \ldots i_{m}}(x) d x_{i_{1}} \wedge \cdots \wedge d x_{i_{m}},
$$

where $x=\left(x_{1}, \ldots, x_{n}\right) \in \mathbb{R}^{n}$, is $G$-invariant if and only if the coefficients $\omega_{i_{1} \ldots i_{p}}$ are constant. Then the differential of the complex $\Omega\left(\mathbb{R}^{n}\right)^{G}$ is trivial.

Evidently the conditions of theorem 2.2 are satisfied for each nonzero $m$-form $\omega$ with constant coefficients on $\mathbb{R}^{n}$ for $p=m-1$. It is easy to check that the sequence of cochains $\varphi_{j, m-j-1} \in C_{\text {diff }}^{j}\left(G, \Omega^{m-j-1}\left(\mathbb{R}^{n}\right)\right)(j=0, \ldots, m-1)$ corresponding to $\omega$ can be defined as follows.

$$
\varphi_{j, m-j-1}\left(a_{1}, \ldots, a_{j}\right)=\frac{(-1)^{j-1}}{m(m-1) \ldots(m-j)} i(x) i\left(a_{j}\right) i\left(a_{j-1}\right) \ldots i\left(a_{1}\right) \omega,
$$

where $a_{k}=\left(a_{k, 1}, \ldots, a_{k, n}\right) \in \mathbb{R}^{n}(k=1, \ldots, j)$ and on the right hand side we consider each $a_{k}$ as a constant vector field on $\mathbb{R}^{n}$ and $x$ as identical vector field on $\mathbb{R}^{n}$. Then we have

$$
\delta^{\prime} \varphi_{m-1,0}\left(a_{1}, \ldots, a_{m}\right)=\frac{1}{m !} i\left(a_{m}\right) i\left(a_{m-1}\right) \ldots i\left(a_{1}\right) \omega,
$$

where $a_{1}, \ldots, a_{m} \in \mathbb{R}^{n}$. 
Take the point $0 \in \mathbb{R}^{n}$ as the cycle $\alpha$. Then $F_{\alpha}: \Omega\left(\mathbb{R}^{n}\right)^{G} \rightarrow \Omega(G)$ is the identity map. By proposition 3.7 the cocycle $c_{a}(\omega)$ is nontrivial in the complex $C^{*}(G, \mathbb{R})$.

\section{Cocycles on groups of Diffeomorphisms}

In this section we indicate nontrival cocycles for groups of diffeomorphisms of a manifold preserving a family of exact forms.

Let $\left(\omega_{i}\right)_{i \in I}$ be a family of smooth differential forms on a manifold $M$. Denote by $\operatorname{Diff}\left(M,\left(\omega_{i}\right)\right)$ the group of diffeomorphisms of $M$ preserving all forms $\omega_{i}$. We consider $\operatorname{Diff}\left(M,\left(\omega_{i}\right)\right)$ as a topological group with respect to $C^{\infty}$-topology or as a infinite-dimensional Lie group if such a structure on $\operatorname{Diff}\left(M,\left(\omega_{i}\right)\right)$ exists. By proposition 4.2 one can suppose that the cocycle $c_{a}\left(\omega_{i}\right)$ is a continuous or differentiable cocycle.

Let $\left(\omega_{i}\right)_{i \in I}$ be a family of nonzero differential forms on $\mathbb{R}^{n}$ with constant coefficients. Put $G=\operatorname{Diff}\left(\mathbb{R}^{n},\left(\omega_{i}\right)\right)$.

Theorem 6.1. Let $\left(\omega_{i}\right)_{i \in I}$ be a family of nonzero differential forms on $\mathbb{R}^{n}$ with constant coefficients such that $\operatorname{deg} \omega_{i}=d_{i}$. Then, for each $i \in I$ and for $0 \in \mathbb{R}^{n}$ as a zero dimensional cycle $\alpha$, the cocycle $c_{a}\left(\omega_{i}\right)$ is defined and nontrivial in the complex $C^{*}(G, \mathbb{R})$.

Proof. Evidently the conditions of theorem 2.2 are satisfied for each form $\omega_{i}$ and $p=$ $m_{i}-1$ and then the cocycle $c_{a}\left(\omega_{i}\right)$ of the complex $C^{*}(G, \mathbb{R})$ is defined. Obviously the group $G$ contains the group $\mathbb{R}^{n}$ acting on $\mathbb{R}^{n}$ by translations. Consider the restriction of the cocycle $c_{a}(\omega)$ to the subgroup $\mathbb{R}^{n}$. By example 5.3 this restriction is nontrivial. Then the cocycle $c_{a}\left(\omega_{i}\right)$ is nontrivial as well.

Corollary 6.2. Let $M$ be a connected manifold such that

$$
H^{1}(M, \mathbb{R})=\cdots=H^{m-1}(M, \mathbb{R})=0,
$$

let $\omega_{0}$ be a nonzero $m$-form on $\mathbb{R}^{n}$ with constant coefficients, and let $\omega_{M}$ be an exact $m$-form on $M$. Consider $\omega=\omega_{0}+\omega_{M}$ as an $m$-form on $\mathbb{R}^{n} \times M$. Then for the group $G=\operatorname{Diff}\left(\mathbb{R}^{n} \times M, \omega\right)$ and a point $x \in \mathbb{R}^{n} \times M$ as a zero dimensional cycle $\alpha$, the cocycle $c_{a}(\omega)$ is defined and nontrivial in the complex $\left.C^{*}\left(R^{n} \times M, \mathbb{R}\right)\right)$.

Proof. Evidently the conditions of theorem 2.2 for the form $\omega$ are satisfied and then the cocycle $c_{a}(\omega)$ of the complex $C^{*}(G, \mathbb{R})$ is defined. Consider the group $\operatorname{Diff}\left(\mathbb{R}^{n}, \omega_{0}\right)$ acting on the first factor of $\mathbb{R}^{n} \times M$ as a subgroup of $G$ and the restriction of the cocycle $c_{a}(\omega)$ to this subgroup. Since the subgroup $\operatorname{Diff}\left(\mathbb{R}^{n}, \omega_{0}\right)$ preserves the form $\omega_{M}$ as a form on $\mathbb{R}^{n} \times M$ by theorem 6.1 this restriction is a nontrivial cocycle. Thus $c_{a}(\omega)$ is a nontrivial cocycle of the complex $C^{*}(G, \mathbb{R})$ as well.

We indicate the following partial case of corollary 6.2 (see also 6]). 
Corollary 6.3. Let $\omega_{0}$ be the standard symplectic 2 -form on $\mathbb{R}^{2 n}$ and let $m=$ $1, \ldots, n$. Let $M$ be a connected manifold such that $2 m \leq \operatorname{dim} M$,

$$
H^{1}(M, \mathbb{R})=\cdots=H^{2 m-1}(M, \mathbb{R})=0,
$$

and $\omega_{M}$ an exact $2 m$-form on a manifold $M$. Consider the $2 m$-form $\omega=\omega_{0}^{m}+\omega_{M}$ on $\mathbb{R}^{2 n} \times M$. Then for the group $G=\operatorname{Diff}\left(\mathbb{R}^{2 n} \times M, \omega\right)$ and a point $x \in \mathbb{R}^{2 n} \times M$ as a zero dimensional cycle $\alpha$, the cocycle $c_{a}(\omega)$ is defined and nontrivial in the complex $\left.C^{*}\left(R^{2 n} \times M, \mathbb{R}\right)\right)$.

Let $M$ be a connected compact oriented manifold with a volume form $v_{M}$ such that $\int_{M} v_{M}=1$.

Theorem 6.4. Let $\left(\omega_{i}\right)_{i \in I}$ be a family of nonzero differential forms on $\mathbb{R}^{n}$ with constant coefficients such that $\operatorname{deg} \omega_{i}=d_{i}$. Consider the family $\left\{\omega_{i} \wedge v_{M}\right\}_{i \in I}$ of forms on $\mathbb{R}^{n} \times M$. For a cycle $\alpha=0 \times M$ of the homology class $a$ on $\mathbb{R}^{n} \times M$ and each $i \in I$ the cocycle $c_{a}\left(\omega_{i} \wedge v_{M}\right)$ on the group $G=\operatorname{Diff}\left(\mathbb{R}^{n} \times M,\left(\omega_{i} \wedge v_{M}\right)\right)$ with values in the trivial $G$-module $\mathbb{R}$ is defined and nontrivial.

Proof. Evidently the conditions of theorem 2.2 for each form $\omega_{i} \wedge v_{M}$ and $p=n-1$ are satisfied. Then the cocycle $c_{a}\left(\omega_{i} \wedge v_{M}\right)$ of the complex $C^{*}(G, \mathbb{R})$ is defined.

Consider the group $\operatorname{Diff}\left(\mathbb{R}^{n},\left(\omega_{i}\right)\right)$ as a subgroup of the group $G$ and the restriction of the cocycle $c_{a}(\omega)$ to this subgroup. Since the subgroup $\operatorname{Diff}\left(\mathbb{R}^{n},\left(\omega_{i}\right)\right)$ preserves the form $v$ as a form on $\mathbb{R}^{n} \times M$, as in the proof of corollary 6.2 we can show that the above restriction is a nontrivial cocycle. Thus the cocycle $c_{a}(\omega)$ is nontrivial as well.

We indicate the following partial case of theorem 6.4

Corollary 6.5. Let $v_{0}$ be the standard volume form on $\mathbb{R}^{n}$. Then for the cycle $a=0 \times M$ the cocycle $c_{a}\left(v_{0} \wedge v_{M}\right)$ on the group $G=\operatorname{Diff}\left(\mathbb{R}^{n} \times M, v_{0} \wedge v_{M}\right)$ is defined and nontrivial in the complex $\left.C^{*}\left(G, v_{0} \wedge v_{M}\right), \mathbb{R}\right)$.

We consider the space $\mathbb{C}^{2 n}$ and a skew-symmetric bilinear form $\omega$ of rank 2 on it. Let $\tilde{\omega}$ be the differential 2-form corresponding to $\omega$ on $\mathbb{C}^{2 n}$ as a complex manifold. By definition the form $\tilde{\omega}$ has constant coefficients. Then $\tilde{\omega}=\tilde{\omega}_{1}+i \tilde{\omega}_{2}$, where $i=\sqrt{-1}, \tilde{\omega}_{1}$ and $\tilde{\omega}_{2}$ are real differential 2-forms with constant coefficients on $\mathbb{C}^{2 n}=\mathbb{R}^{4 n}$ as a real $4 n$-dimensional manifold.

Similarly, consider the space $\mathbb{C}^{n}$, a sqew-symmetric $n$-form $v$ of maximal rank on it, and the corresponding differential $n$-form $\tilde{v}$ on $\mathbb{C}^{n}$. Consider the real differentiable $n$-forms $\tilde{v}_{1}$ and $\tilde{v}_{2}$ on $\mathbb{C}^{n}=\mathbb{R}^{2 n}$ as a real $2 n$-dimensional manifold defined by an equality $\tilde{v}=\tilde{v}_{1}+i \tilde{v}_{2}$.

In both cases above we can apply theorem 6.1 to the forms $\tilde{\omega}_{1}, \tilde{\omega}_{2}, \tilde{v}_{1}$ and $\tilde{v}_{2}$. We leave to the reader to define the corresponding cocycles on the group of diffeomorphisms preserving the forms $\tilde{\omega}$ and $\tilde{v}$ and to formulate the statement similar to those of corollaries 6.2 and 6.4 


\section{REFERENCES}

[1] H. Cartan, S. Eilenberg, Homological algebra, Princeton, Princeton University Press, 1956.

[2] W.T. van Est, Group cohomology and Lie algebra cohomology in Lie groups. I, II, Indagationes Mathematicae, 15 (1953), 484-492; 493-504.

[3] A. Guichardet, Cohomologie des groupes topologiques et des algèbres de Lie, Cedic/Nathan, Paris, 1980.

[4] G.P. Hochschild, G.D. Mostow, Cohomology of Lie algebras, Ann. Math, 57 (1953), no 3, 591-603.

[5] M.V. Losik, Characteristic classes of transformation groups, Diff. Geom. Appl., 3 (1993) 205-218.

[6] M. Losik, P.W. Michor Extensions for a group of diffeomorphisms of a manifold preserving an exact 2-form, arXiv:math.GR/0410100

[7] G.D. Mostow, Cohomology on topological groups and solvmanifolds, Ann. Math., 73 (1961), $20-48$.

[8] V.P. Palamodov, The complex Dolbeault on a Stein manifold splits in positive dimensions, Mat. Sb. (N.S.) 88(130),no. 2(6) (1972), 287-315 (Russian).

[9] G. de Rham , Variétés différentiableles, Paris, Hermann, 1955.

M. Losik: Saratov State University, Astrakhanskaya, 83, 410026 Saratov, Russia.

E-mail address: LosikMV@info.sgu.ru

P. W. Michor: Fakultät für Mathematik, Universität Wien, Nordbergstrasse 15, A1090 Wien, Austria; and: Erwin Schrödinger Institute of Mathematical Physics, Boltzmanngasse 9, A-1090 Wien, Austria.

E-mail address: Peter.Michor@esi.ac.at 